Academic Department of Rehabilitation Medicine, Leeds Institute of Rheumatic and

Musculoskeletal Medicine, University of Leeds, Leeds, UK

2 National Demonstration Centre in Rehabilitation Medicine, Leeds Teaching Hospitals NHS Trust, Leeds, UK

3 Central and North West London NHS Foundation Trust, London, UK

4 Imperial College School of Medicine, London, UK

Correspondence to: M Sivan M.Sivan@leeds.ac.uk

Cite this as: BMJ2020;371:m4938 http://dx.doi.org/10.1136/bmj.m4938 Published: 23 December 2020

\section{NICE guideline on long covid}

\section{Research must be done urgently to fill the many gaps in this new "living guideline" Manoj Sivan, ${ }^{1,2}$ Sharon Taylor ${ }^{3,4}$}

The covid-19 pandemic has killed over 1.6 million people worldwide, ${ }^{1}$ caused the worst healthcare crisis of this century, and put a huge dent in our economies. The magnitude of the population still struggling with symptoms four weeks after their acute illness-commonly called "long covid"-is becoming obvious and demands urgent prioritisation to prevent a further blow to health systems and the healthcare workforce.

Long covid is thought to occur in approximately $10 \%$ of people infected, ${ }^{23}$ so there are likely more than 5 million people affected globally. The National Institute for Health and Care Excellence (NICE), the Scottish Intercollegiate Guidelines Network, and the Royal College of General Practitioners have developed a rapid guideline for managing the long term effects of covid-194 to assist long covid services being set up in the $\mathrm{NHS}^{5}$ and elsewhere. A lack of systematic research means that the current guideline is necessarily preliminary, but it will be updated regularly as new evidence emerges-becoming a "living guideline." This approach is essential as the current guideline lacks important detail, including a comprehensive list of organ complications seen in patients with long covid, the investigations required, and specific interventions for these complications.

\section{Standardised terms}

The guideline defines long covid as "signs and symptoms that develop during or following an infection consistent with covid-19 and which continue for more than four weeks and are not explained by an alternative diagnosis." ${ }^{4}$ Given that we are beginning to understand the underlying immunological and organ specific effects of SARS-CoV-2, 67 it might have been better to define it as "signs and symptoms that continue for more than four weeks and can be attributed to covid-19 infection." This definition would include all the post-acute medical complications of covid-19 under one unified definition rather than making long covid a vague diagnosis of exclusion.

NICE recommends using the term "post-covid syndrome” from 12 weeks after infection. But no evidence exists of any particular physiological changes (that predict chronicity) at 12 weeks, so it would be preferable to use the term long covid for symptoms of any duration beyond four weeks, as is strongly advocated by people with lived experience of this condition. ${ }^{8}$ Using the prefix "post" implies that acute infection and any active disease process ave resolved, which is currently unknown.

\section{Comprehensive assessment}

The guideline rightly includes people with suspected covid-19 infection without requiring a positive antigen or antibody test. Any other approach risks missing the many thousands of people who did not have access to testing in the early phases of the pandemic. The recommendations emphasise comprehensive assessment by a multidisciplinary team and support the use of virtual methods of assessment, including screening questionnaires such as C19-YRS (Yorkshire Rehabilitation Screen). ${ }^{9}$ They also warn clinicians not to rely on questionnaires alone and to undertake additional thorough assessments.

The guidance mentions screening blood tests (such as full blood count; clotting profile; renal, liver, and thyroid functions; and $\mathrm{C}$ reactive protein) and tests of both cardiac and lung function to capture reversible abnormalities, but it lacks detail on the management of serious life threatening complications such as a hypercoagulable state. ${ }^{10}$ The next update should prioritise how to screen for, diagnose, and manage medical complications ${ }^{11}$ reported in patients with long covid including silent desaturations; cardiac, respiratory, renal, hepatic, gastrointestinal, and neurological abnormalities; endocrine problems; autonomic dysregulation and postural tachycardia; and mast cell disorder. Missing these complications could result in serious adverse outcomes for patients.

One stop multidisciplinary clinics are recommended, led by a doctor with relevant specialist skills and experience. NHS England has also emphasised the importance of multidisciplinary assessment and diagnostics being available in long covid clinics to avoid multiple referrals to different specialists. ${ }^{5}$ Clearer guidance on the optimal composition of multidisciplinary teams would have been helpful. Respiratory physicians, cardiologists, neurologists, general physicians (from primary care or rehabilitation medicine), neuropsychologists or neuropsychiatrists, physiotherapists, occupational therapists, speech and language therapists, and dieticians may all be required.

\section{Interventions}

Shared decision making is appropriately emphasised in the setting of goals and the formulation of personalised management plans and care plans. The guidance lacks detail on potentially helpful rehabilitation interventions such as breathing techniques, psychological interventions (such as cognitive behaviour therapy), cognitive training (such as memory training), and occupational rehabilitation, perhaps understandably given the current paucity of supporting evidence. The dangers of exercise in some patients, such as those with undiagnosed acute pericarditis or myocarditis, highlight the need for a personalised approach. ${ }^{12}$ All these aspects of care should be prioritised in future updates. 
Research on the underlying pathophysiology of long covid is now urgent, including identifying the immunological, inflammatory, genetic, metabolic, and psychological correlates, to better understand symptom development. The NICE guideline rightly recommends further research to characterise common clusters of symptoms (phenotypes), risk factors, prognostic markers, natural history, and trajectory of long covid, and to identify effective interventions for all age groups.

The need to tackle health inequalities in care for people with long covid-such as those with mental health conditions, learning disabilities, or specific cultural needs, or where there are language barriers-is emphasised in the guidance. The key priority is to understand the mechanisms causing long covid, so that organ complications might be managed early to prevent long term symptoms and serious adverse consequences and to enable individuals to return to their normal lives as soon as possible.

Provenance and peer review: Commissioned, not peer reviewed.

Competing interests: We have read and understood BMJ policy on declaration of interests and declare the following interests: MS and colleagues developed the C19-YRS (Yorkshire Rehabilitation Scale) and are involved in delivering long covid care in the Leeds multidisciplinary long covid assessment and management service. ST has ongoing organ complications of covid-19 and is a member of the UK doctors \#longcovid group. Both authors were part of a stakeholder group that commented on the NICE draft guidance.

1 World Health Organization. WHO coronavirus disease (covid-19) dashboard. https://covid19.who.int/

2 Greenhalgh T, Knight M, A'Court C, Buxton M, Husain L. Management of post-acute covid-19 in primary care. BMJ2020;370:m3026. doi: 10.1136/bmj.m3026 pmid: 32784198

3 Sudre CH, Murray B, Varsavsky T, et al. Attributes and predictors of long-covid: analysis of covid cases and their symptoms collected by the Covid Symptoms Study app. MedRxiv [preprint]. https://www.medrxiv.org/content/10.1101/2020.10.19.20214494v1

4 NICE. Covid-19 rapid guideline: managing the long-term effects of covid-19. https://www.nice.org.uk/guidance/ng188/chapter/4-Planning-care

$5 \quad$ NHS England. National guidance for post-covid syndrome assessment clinics. Nov 2020. https://www.england.nhs.uk/coronavirus/publication/national-guidance-for-post-covid-syndromeassessment-clinics/

6 Manolis AS, Manolis AA, Manolis TA, Apostolopoulos EJ, Papatheou D, Melita H. Covid-19 infection and cardiac arrhythmias. Trends Cardiovasc Med 2020;30:451-60. doi: 10.1016/j.tcm.2020.08.002 pmid: 32814095

7 Dennis A, Wamil M, Kapur S, et al. A multi-organ impairment in low-risk individuals with long COVID. MedRxiv [preprint]https://www.medrxiv.org/content/10.1101/2020.10.14.20212555v1

8 Callard F, Perego E. How and why patients made long covid. Soc Sci Med 2020;113426:113426. doi: 10.1016/j.socscimed.2020.113426. pmid: 33199035

9 Sivan M, Halpin SJ, Gee J. Assessing long-term rehabilitation needs in covid-19 survivors using a telephone screening tool (C19-YRS tool). Adv Clin Neurosci Rehabil 2020;19:14-7doi: 10.47795/NELE5960 .

10 Zhai Z, Li C, Chen Y, etalPrevention Treatment of VTE Associated with COVID-19 Infection Consensus Statement Group. Prevention and treatment of venous thromboembolism associated with coronavirus disease 2019 infection: a consensus statement before guidelines. Thromb Haemost 2020;120:937-48. doi: 10.1055/s-0040-1710019 pmid: 32316065

11 Rahmani H, Davoudi-Monfared E, Nourian A, etal. Comparing outcomes of hospitalized patients with moderate and severe COVID-19 following treatment with hydroxychloroquine plus atazanavir/ritonavir. Daru2020;28:625-34. doi: 10.1007/s40199-020-00369-2 pmid: 32857301

12 Phelan D, Kim JH, Elliott MD, etal. Screening of potential cardiac involvement in competitive athletes recovering from covid-19: An expert consensus statement. JACC Cardiovasc Imaging 2020;13:2635-52. doi: 10.1016/j.jcmg.2020.10.005 pmid: 33303102 